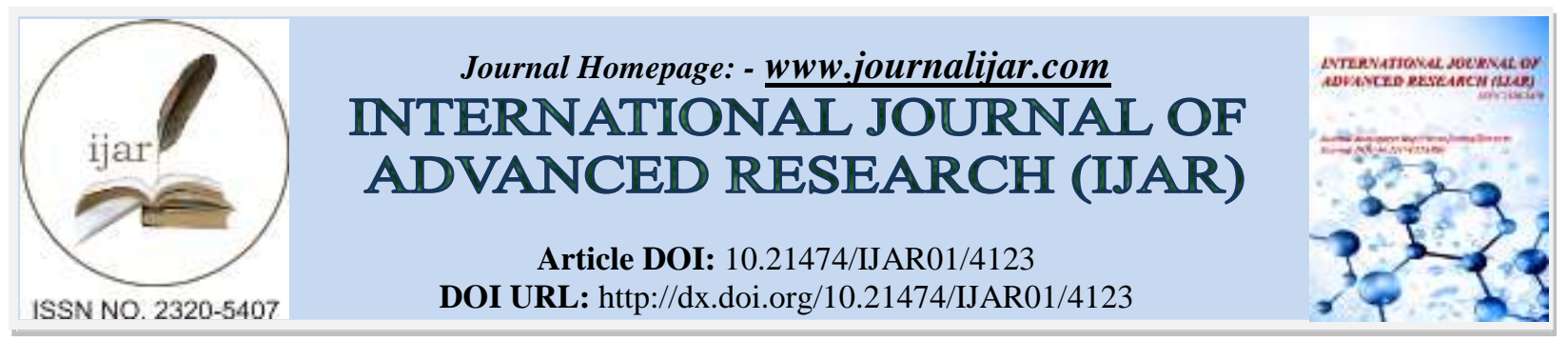

RESEARCH ARTICLE

\title{
STOCHASTIC PROCESS DESCRIPTION VIA INTERPRETATION TO A SINGLE MOLECULAR ROTOR.
}

\section{Wan Qashishah Akmal Wan Razali ${ }^{1}$, Shahidan Radiman ${ }^{1}$, Hishamuddin Zainuddin ${ }^{2}$ and Siti Norafidah Mohd Ramli'.}

1. School of Applied Physics, Faculty of Science and Technology, National University of Malaysia (UKM), 43600 Bangi, Selangor, Malaysia.

2. Institute for Mathematical Research (INSPEM) Universiti Putra Malaysia (UPM), 43400 Serdang, Selangor, Malaysia.

3. School of Mathematics, Faculty of Science and Technology, National University of Malaysia (UKM), 43600 Bangi, Selangor, Malaysia.

\section{Manuscript Info}

-.......................

Manuscript History

Received: 02 March 2017

Final Accepted: 05 April 2017

Published: May 2017

Key words:-

Molecular motor, Bohmian theory, stochastic force, dissipation.

\begin{abstract}
Stochastic processes has been described using Bohmian quantum mechanics by various researchers for dissipative quantum system in various applications. The Madelung quantum hydrodynamic picture in reinterpreting the stochastic process was found to be useful in describing microscopic molecular systems. In this paper, we illustrated a single molecular rotor by means of the Madelung-Bohm formalism as the quantum fluctuations corresponding to quantum stochastic force. We extended the modified Liouville equation in de Broglie-Bohm theory to associate with the mechanism of decoherence in the molecular rotor that used the motive power to control and create the motion in a non-stationary-state superposition. Thus, the interaction of the molecular rotor with the reservoir would provide the thermal fluctuation effect in the surrounding which was accounted for by two random forces. A new application of Bohmian mechanics in a single molecular rotor was proposed with both dissipative potential and quantum potential. The two random forces that described the random rotational fluctuations were the friction term and diffusion behavior of the Brownian ratchet motion in the rotor. It was explicitly shown that stochastic process in the rotor was equivalent to the quantum potential in the Bohmian theory.
\end{abstract}

Copy Right, IJAR, 2017,. All rights reserved.

\section{Introduction:-}

Recently, the de Broglie-Bohm causal interpretation of quantum theory has been used in many applications of manybody systems (Benseny et al., 2014). In generating observed quantum behavior of a system, the ideas of the classical deterministic theory with dissipation mechanism and the theorem on stable trajectories in Bohmian dynamics had been introduced (Eigorn and Rusov, 2014). However, the route to describe Bohmian mechanics in hydrodynamic fashion has been developed by Mandelung-Bohm formalism (Wyatt, 2005; Sonego, 1991). 
It is also known that the inclusion of hydrodynamic interaction in molecular environment has played a crucial role in describing the motility of molecular machine such as kinesin motor dynamics (Goldtzvik et al., 2016). In fact, the specific interaction and molecular fluctuations cannot be taken into account within continuum hydrodynamics model, but they can be incorporated with the environment due to coarse grained molecular dynamics (Cressman et al., 2008; Joachim and Ratner, 2005).

Molecular machine such as molecular rotor has been well described for unidirectional rotation by Schrödinger equation with decoherence and classical motion. This theory has been applied to a self-assembling molecule, which is a molecular motor that interacts with its surface within a given motive power as shown in the Figure 1 (Joachim and Gimzewski, 2001).

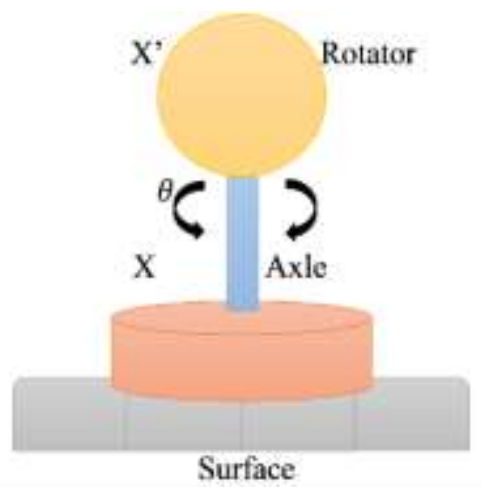

Figure 1:- Molecular rotor and its surface.

It consists of two parts which are a rotator on the axle and a stator that fixes the axis in space (Joachim and Gimzewski, 2001; Kottas et al., 2005). According to some findings, the rotation of molecular rotor may excurse to opposite motion occasionally within the background random Brownian noise. By triggering external work, these can lead to the unidirectional motion with ratchet mechanism like a rolling wheel either in clockwise or anti-clockwise direction (Cressman et al., 2008; Joachim and Gimzewski, 2001).

The random force that effectively acts on the rotor is obtained from the dynamical equation and the standard probability conservation equation. Given the random interaction of the reservoir, the driven force and rotational potential have been designed to contribute as a working paradigm even though their operation is still questionable as it depends on the profile of asymmetric potential and the noise spectrum in a subtle equilibrium (Joachim and Gimzewski, 2001; Kottas et al., 2005).

The dynamics of the rotor dissipative systems that includes diffusion, decoherence and non-equilibrium phenomena, allows the exchange of the energy with the environment. Besides that, such supramolecular assembly of the nanoscale machine has the ability to transform surface thermal energy into fluctuations of random rotational motion. In this regard, the time-dependent Hamiltonian is used with the inclusion of the effect of the environment. For instance, the coupling of the system to a large number of environmental degrees of freedom in the system-plusreservoir can be modelled by harmonic oscillators (Chou and Wyatt, 2007).

The rotor part of molecular motor should be considered as a semi-classical rotating motion in a non-stationary state of an isolated quantum system which can be achieved in two ways in order to ensure a permanent quantum motion. It can either be by controlling the interaction of the rotatory motor with the environment or by controlling the quantum state carefully via the time evolution of the wave packet. The presence of reservoirs is necessary as the energy of rotation rely on this mixing with the environment and the quantum character are generated by the merging of eigenstates with continuum states of the environment. The environment can also be considered as the interaction of rotor and reservoir that are driven by a large number of degrees of freedom of a system or another source such as microwave and surface interaction (Joachim and Gimzewski, 2001).

The motion of the rotor essentially is governed classical mechanically. Thus, we consider the connection between motion and forces which are feasible by including the speed and the coordinates of the nanomachine. Down to nano scale, the Schrödinger equation appears in the ensemble average correspondence between force and quantum eigenstates of a nanomachine. However, since the nanomachine is in unidirectional rotation at a macroscopic scale, 
such correspondence is not very intuitive as the superposition of eigenstates for the motion does not all times produce the exact solution that satisfy the classical equation (Joachim and Gimzewski, 2001; Tang et al., 1997).

Bohmian mechanics is most convenient framework to incorporate the Newtonian force with an additional force describing quantum fluctuations (Nauenberg, 2014; Tsekov, 2009). In (Tsekov 2012), the pilot wave theory has been considered as an approximation to a mean-field theory of the quantum dynamics that consist of stochastic behavior of the vacuum fluctuations. In the present example, this can be made in agreement with the random rotational fluctuations in the single molecular rotor due the interaction of the reservoirs. In order to interpret the statistical influence of the environment and thermal diffusion, the random potential and the dissipative potential are incorporated into the time-dependent Schrödinger equation (TDSE) (Joachim and Gimzewski, 2001; Chou and Wyatt, 2007). Hence, the stochastic process that contains the nature of the non-stationary states can be described by the Bohmian quantum potential.

This paper is divided into the following. In theoretical approach section, we discuss our approach on the extension from the modified Liouville equation in the Bohmian interpretation (Eigorn and Rusov, 2014). Next, we apply this theoretical approach to a single molecular rotor. Then, we describe the dissipation mechanism underlying in the quantum fluctuations of the rotor by introducing quantum Brownian motion which is a continuation from the theoretical approach.

\section{Theoretical Approach:-}

We introduce the modified Liouville equation in the de Broglie-Bohm theory as in equation (1), which is also wellknown as the continuity equation (Eigorn and Rusov, 2014):

$\frac{d f_{N}}{d t}=\left.\frac{\partial f_{N}}{\partial t}\right|_{r_{k}, v_{k}}+\left.\sum_{i=1}^{N} v_{i} \frac{\partial f_{N}}{\partial r_{i}}\right|_{v_{k}, t}+\left.\frac{1}{m} \sum_{i=1}^{N} F_{i}\left(r_{k}, t\right) \frac{d f_{N}}{\partial v_{i}}\right|_{r_{k}, t}=0$

Whereby $v_{k}(t)$ and $r_{k}(t)$ are the velocity and the radius-vector of the $k$-th particle respectively, while $m$ is the mass of a single particle. The function $f_{N}\left(r_{k}, v_{k}, t\right)$ here is represented as the probability density in the phase space. The system of $N$ identical particles obeys Newton's second law;

$\frac{d r_{i}}{d t}=v_{i}, m \frac{d v_{i}}{d t}=F_{i}\left(r_{k}, t\right)=-\frac{\partial U}{\partial r_{i}}$

According to Bohmian mechanics, the potential energy, $U$ can be divided into the classical potential, $U_{c l}$ and the quantum part, $Q$ :

$U\left(r_{k}, t\right)=U_{c l}\left(r_{k}, t\right)+Q\left(r_{k}, t\right)$

in which quantum potential is given by

$Q\left(r_{k}, t\right)=-\frac{\hbar^{2}}{2 m} \sum_{j=1}^{N} \frac{\Delta_{j} A}{A}=-\frac{\hbar^{2}}{2 m} \sum_{j=1}^{N} \frac{\Delta_{j} \sqrt{f_{N}\left(r_{k}, v_{k}, t\right) \prod_{l=1}^{N} d v_{l}}}{\sqrt{f_{N}\left(r_{k}, v_{k}, t\right) \prod_{l=1}^{N} d v_{l}}}$

Where $A$ is amplitude of $N$ particles. By substituting equations (2), (3) and (4) into (1), one has

$\frac{d f_{N}}{d t}=\left.\frac{\partial f_{N}}{\partial t}\right|_{r_{k}, v_{k}}+\left.\sum_{i=1}^{N} v_{i} \frac{\partial f_{N}}{\partial r_{i}}\right|_{v_{k}, t}$

$+-\left.\left.\frac{1}{m} \sum_{i=1}^{N} \frac{\partial U_{c l}}{\partial r_{i}}\right|_{t} \frac{\partial f_{N}}{\partial v_{i}}\right|_{r_{k}, t}+-\left.\frac{\hbar^{2}}{2 m} \sum_{i=1}^{N} \frac{\partial}{\partial r_{i}}\left(\sum_{j=1}^{N} \frac{\Delta_{j} \sqrt{f_{N}\left(r_{k}, v_{k}, t\right) \prod_{l=1}^{N} d v_{i}}}{\sqrt{f_{N}\left(r_{k}, v_{k}, t\right) \prod_{l=1}^{N} d v_{i}}}\right) \frac{\partial f_{N}}{\partial v_{i}}\right|_{r_{k}, t}=0$

This equation carries all the information about the system but it requires some simplification. The last term of quantum nature comes from equation (4) implies that nonlinearity dependence on $N$-particle distribution function $f_{N}\left(r_{k}, v_{k}, t\right)$ with the order of $\hbar^{2}$. Since $d f / d t=0$, so that $f$ is constant along a path of phase space. In this study, the theory can be associated with the molecular machine with rotatory motion.

\section{Application to Single Molecular Rotor:-}

The use of decoherence and classical motion in a single molecular rotor has been explained in (Joachim and Gimzewski, 2001) using TDSE of the rotor and its surface. We now reinterpret these ideas using Bohmian mechanics.

We use both classical mechanics (microscale) and quantum mechanics (nanoscale) to describe the mechanical rotation of the nanomachine. Newton's equation of motion describes the motion with torsional forces in which the coordinates and speed of each part of the nanomachine relative to the origin is given by; 
$F(\theta, t)=I \frac{d^{2}}{d t^{2}}\langle\theta(t)\rangle=-\frac{d V\langle\theta(t)\rangle}{d\langle\theta(t)\rangle}$

where $V(\theta)$ is an external periodic potential energy profile whereby the rotation axis is the $X-X^{\prime}$ axis in order to find the multiple equilibrium positions of the molecule (stations), $I$ is the moment of inertia of the rotor and $\theta$ is the angular position (Joachim and Gimzewski, 2001; Kottas et al., 2005; Browne and Feringa, 2006). The kind of motion is illustrated by the Figure 1 .

The average angle $\theta(t)$ with a system of $N$ molecules of the rotatory motor can be written as $\theta(t)=1 / N \sum_{k}\left\langle\theta_{k}(t)\right\rangle$. More intuitively, the angular variable in equation (6) transforms according to TDSE with average value $\langle\theta(t)\rangle=$ $\langle\chi(\theta, t)|\Theta| \chi(\theta, t)\rangle$ i.e. as the angular motion $\Theta$ of angular state $\chi(\theta, t)$. The quantum state of the molecular rotor associates with it a rotational wave packet which relies on a material of the nanoscale object before the dilution of wave packet occurs, obtained using the Ehrenfest theorem (Joachim and Gimzewski, 2001):

$I \frac{d^{2}}{d t^{2}}\langle\theta(t)\rangle=-\left\langle\chi(\theta, t)\left|\frac{d U(\theta)}{d \theta}\right| \chi(\theta, t)\right\rangle$

For the initial wave packet in a $\chi(\theta, 0)$ non-stationary state, we write it as $\chi(\theta, 0)=\sum_{n} C_{n}(0) \chi_{n}(\theta)$

whereby the complex-valued coefficient $C_{n}$ and the eigenstates $\chi_{n}(\theta)$ are selected to make (7) comparable to (6). Hence, by finding a set of complex-valued numbers $C_{n}$, the motive power at $(\theta, 0)$ can be obtained (Joachim and Gimzewski, 2001; Joachim and Rapenne, 2015).

From this, the unidirectional rotation of nanomachine will be free if the eigenstates $\chi_{n}(\theta)$ interact with sources of energies or environment (reservoir) satisfying the second law of thermodynamics. The rotor and the surface system can be described by a single wave function, $\Psi$ which is maintained by a reservoir at a given temperature, $\mathrm{T}$ as; $\Psi\left(\theta, r_{k}, t\right)=\sum_{n} \sum_{P_{k}} A_{n, P_{k}}(t) \chi_{n}(\theta) g_{p}\left(r_{k}\right) \quad(9)$

where $\chi_{n}(\theta)$ is the rotor state which is taken the tensor product with the quantum state $g_{p}\left(r_{k}\right)$ of the surface system.

Essentially, the phase coherence of any wavelength can be destroyed by the interaction of a quantum state with the environment leading to more semi-classical-like behavior. For this purpose, the Madelung polar form of the wave function is used to employ the motion of rotor itself with direct extraction (Joachim and Gimzewski, 2001):

$\Psi\left(\theta, r_{k}, t\right)=\phi\left(\theta, r_{k}, t\right) \exp \frac{i}{\hbar} S_{T}\left(\theta, r_{k}, t\right)$

Here, $S_{\mathrm{T}}$ is the total action of both the nanomachine and its surface and $\phi$ is amplitude of the wavefunction. The probability density, $\rho$ corresponds to the square modulus of the $N$-particles (total particles represented by index $k$ ) of the amplitude of the wave function in the polar form.

$\rho\left(\theta, r_{k}, t\right)=\left|\phi\left(\theta, r_{k}, t\right)\right|^{2} \quad(11)$

The polar form equation (10) of the wave function can be substituted into TDSE, giving;

$\hbar \frac{\partial}{\partial t} \Psi\left(\theta, r_{k}, t\right)=\left[-\frac{\hbar \partial^{2}}{2 I \partial \theta^{2}}-\sum_{k} \frac{-\hbar^{2}}{2 m k} \Delta_{r_{k}}+V\left(\theta, r_{k}, t\right)\right] \Psi\left(\theta, r_{k}, t\right)$

The first and second terms on the right side of the equation represent the energies with angular motion of molecular rotor itself in association to its surface (Joachim and Gimzewski, 2001).

Based on the de Broglie theory, the potential energy can be divided into classical part, $U_{c l}$ and quantum part, $Q$ (Eigorn and Rusov, 2014; Tsekov, 2009, 2012; Crowell, 2005)i.e.

$V\left(\theta, r_{k}, t\right)=U_{c l}\left(\theta, r_{k}, t\right)+Q\left(\theta, r_{k}, t\right)$

The quantum potential $Q\left(\theta, r_{k}, t\right)$ act as wave guides that piloted the trajectories of the particles in Bohmian theory which are dependent on the whole wavefunction. By incorporating the probability density in phase space function in the theoretical approach with this nanorotor, the normalized probability density can be written as

$\int f_{N}\left(\theta, r_{1}, \ldots, r_{k}, t\right) \prod_{n=1}^{N} d \theta d \tau_{n}=\int \rho\left(\theta, r_{1}, \ldots, r_{k}, t\right) \prod_{n=1}^{N} d \theta d \tau_{n}=1$

By substituting (14) into the quantum potential in (4), we obtain the quantum potential in term of rotatory motion as

$Q\left(\theta, r_{k}, t\right)=-\frac{\hbar^{2}}{2 m^{2}} \sum_{j=1}^{N}\left(\frac{\Delta_{j} \sqrt{\int \rho\left(\theta, r_{1}, \ldots, r_{k}, t\right) \prod_{n=1}^{N} d \theta d \tau_{n}}}{\sqrt{\int \rho\left(\theta, r_{1}, \ldots, r_{k}, t\right) \prod_{n=1}^{N} d \theta d \tau_{n}}}\right)$

It is obvious that the quantum force becomes negligible if only the particle's mass $m$ is large enough. The quantum behaviour in the de Broglie theory simply adds the quantum potential $Q$ in (13) which is dependent on the probability density $\rho\left(\theta, r_{k}, t\right)$ and in turn obtain trajectories at a given angle $\theta$, radius $r_{k}$ and time $t$. 
In this case, the hydrodynamic polar form of the wave function in Schrödinger equation is based on ansatz with rotatory variables (Garashchukand Rassolov, 2002) rewritten as;

$$
\Psi\left(\theta, r_{k}, t\right)=\sqrt{\rho\left(\theta, r_{k}, t\right)} \exp \frac{i}{\hbar} S_{T}\left(\theta, r_{k}, t\right)
$$

Where amplitude or density $\sqrt{\rho\left(\theta, r_{k}, t\right)}$ and phase $S_{T}\left(\theta, r_{k}, t\right)$ representing the total action are both real functions. A set of dynamic equations and the standard probability conservation equation is obtained by substituting equation (9) by (16) in (12) (Joachim and Gimzewski, 2001).

Based on the continuity equation in (1), the unidirectional rotatory motion becomes equivalent to an equation;

$\frac{d \rho}{d t}=\left.\frac{\partial \rho}{\partial t}\right|_{\theta, \omega_{k}}+\left.\sum_{i=1}^{N} \omega_{i} \frac{\partial \rho}{\partial \theta_{i}}\right|_{\omega_{k}, t}+\left.\frac{1}{m} \sum_{i=1}^{N} F_{i}(\theta, t) \frac{\partial \rho}{\partial \omega_{i}}\right|_{\theta, t}=0$

Where the angular velocity parameters are defined as $\omega=\langle\chi(\theta, t)|\dot{\theta}| \chi(\theta, t)\rangle$. Then, substituting (6), (13) and (15) into (17) gives

$$
\begin{aligned}
& \frac{d \rho}{d t}=\left.\frac{\partial \rho}{\partial t}\right|_{\theta, \omega_{k}}+\left.\sum_{i=1}^{N} \omega_{i} \frac{\partial \rho}{\partial \theta_{i}}\right|_{\omega_{k}, t}-\left.\left.\frac{1}{m} \sum_{i=1}^{N} \frac{\partial U_{c l}}{\partial \theta}\right|_{t} \frac{\partial \rho}{\partial \omega_{i}}\right|_{\theta, t}+- \\
& \left.\frac{\hbar^{2}}{2 m} \sum_{i=1}^{N} \frac{\partial}{\partial r_{i}}\left(\sum_{j=1}^{N} \frac{\Delta_{j} \sqrt{\int \rho\left(\theta, r_{1}, \ldots, r_{k}, t\right) \prod_{n=1}^{N} d \theta d \tau_{n}}}{\sqrt{\int \rho\left(\theta, r_{1}, \ldots, r_{k}, t\right) \prod_{n=1}^{N} d \theta d \tau_{n}}}\right) \frac{\partial \rho}{\partial \omega_{i}}\right|_{\theta, t}=0
\end{aligned}
$$

Here, the third term in the torsional force is once again shown in terms of classical and quantum parts of potential energy. This equation is extended from the modified Liouville equation in equation (1). However, quantum potential in the rotor machine is considered to engage Brownian rotation motion and describe its rotational fluctuations. The formalism of the quantum potential will be explained in the next section in the presence of an environmental setting.

\section{Quantum Fluctuations:-}

We note that the Madelung-Bohm formalism allows the Bohmian mechanics to ascribe hydrodynamical equations in providing the statistical information of the motion of the particle (Wyatt, 2005; Sonego, 1991). Additionally, the de Broglie-Bohm pilot-wave mechanics is a way to illustrate dissipative quantum system by Newtonian equations which is explicitly stochastic. Both dissipative potential and quantum stochastic force that represent the quantum Brownian motion are simultaneously acting by a dissipative quantum Newtonian equation (Joachim and Gimzewski, 2001; Kottas et al., 2005). In the case of rotational rotor, we derive the following stochastic force equation:

$F^{*}(\theta, t)=I \frac{\delta \omega}{\delta t}=-\mu \frac{\delta \theta}{\delta t}-\nabla(U+Q)$

Where $\mu$ is the friction coefficient, $I$ is inertial moment and $\omega$ is angular velocity while $\theta$ is angle of rotatory motion. The quantum stochastic force $\mathrm{Q}$ is purely from vacuum fluctuations that underlie the Ehrenfest theorem (Nauenberg, 2014). Now, let $U=U_{c l}$ for classical potential and hence

$F^{*}(\theta, t)=I \frac{\delta \omega}{\delta t}=-\mu \frac{\delta \theta}{\delta t}-\nabla\left(U_{c l}+Q\right)$

The original torsional force acting on the molecular rotor can be written as (Joachim and Gimzewski, 2001):

$F(\theta, t)=I \frac{\delta \omega(t)}{\delta t}=-\frac{d}{d \theta} V_{e f f}(\theta)-\mu \omega(t)+\xi(T, t)$

By comparing $F^{*}(\theta, t)=F(\theta, t)$ (equations (20) and (21)), we find

$-\frac{d}{d \theta} V_{e f f}(\theta)-\mu \omega(t)+\xi(T, t)=-\mu \frac{\delta \theta}{\delta t}-\nabla\left(U_{c l}+Q\right)$

It is obvious that;

$\nabla U_{c l}=\frac{d}{d \theta} V_{e f f}(\theta), \quad \mu \omega(t)=\mu \frac{\delta \theta}{\delta t}, \quad \xi=-\nabla Q$

The effective potential energy $V_{e f f}(t)$ is related to classical potential force, $U_{c l}$ with a frictional term $\mu$ specifying the change of angular velocity, $\omega(t)=\delta \theta / \delta t$ which can be treated as dissipative force, while the stochastic process 
$\xi$ is equivalent to quantum stochastic force $-\nabla Q$ due to interaction of the environment. Therefore, we rewrite equation (15) as

$\xi(T, t)=-\nabla Q=\frac{\hbar^{2}}{2 m \sqrt{\rho\left(\theta, r_{k}, t\right)}} \frac{\partial^{2} \sqrt{\rho\left(\theta, r_{k}, t\right)}}{\partial r^{2}}$

In fact, the quantum potential $\mathrm{Q}$ here is a thermal chemical potential which corresponds to a dissipative thermal continuity equation in (1). Finally, we deduce that the rotational equation of torsional force in the rotor is absolutely consistent with the modified Liouville-Bohmian theory in (Eigorn and Rusov, 2014).

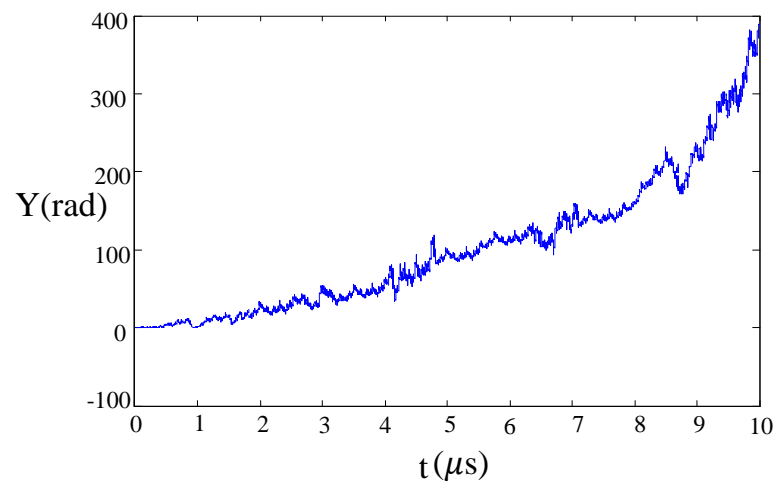

Figure 2:- Graph of $\mathrm{Y}(\mathrm{rad})$ against $\mathrm{t}(\mu \mathrm{s})$

Figure 2 shows the verification of a trajectory of stochastic process - quantum potential in the rotor molecule. Y(rad) axis here is represented as a rotatory trajectory in the rotor against time (in microseconds). The stochastic mechanics that existed in the rotor (real experiment) is convenient and most compatible with our developing theory to elucidate the trajectories of stochastic process in the rotor molecule (Schönborn et al., 2009; Echeverria and Joachim, 2015).

The rotor molecule design has almost the same working principle and concept even though they have different nanomaterials. The thermal motion around the rotor single bond has unidirectional rotation due to temporary formation of a chemical bond between stator and rotator part. A ground state wavepacket pumped into the excited state as the rotation is started. This rotation ultimately change into remaining random motion due to intervention of environment that have been converted by dissipative energy (Joachim and Gimzewski, 2001). By considering the driving force of the rotational potential introduced by us, Brownian motion of the rotor may be associated with heat bath under such working principle.

\section{Conclusion:-}

This article showed that the stochastic process in molecular rotor can be represented by quantum stochastic force via Bohmian Mechanics. We found that the stochastic process, $\xi$ is equivalent to quantum potential Q in the de BroglieBohm theory which describe the diffusion behavior, while another random force that consists of frictional term $\mu$ is found to be the dissipative potential of the rotor system. Therefore, our finding is consistent with fluctuation theorem in nonequilibrium thermodynamics of the motor as well as capable to illustrate the Brownian ratchet motion. For future work, this research can be extended to linear molecular motor such as kinesin, myosin and dynein upon obtaining the master equation of the quantum force to describe their fluctuations behavior. However, it needs to focus on several key advances of many current issues in the field and highlight future challenges for the field of molecular rotors and motors.

\section{Acknowledgement:-}

The authors gratefully acknowledge National University of Malaysia, UKM (Grant no. : FRGS/1/2014/SG05/UKM/01/1) for financial support. 


\section{References:-}

1. Benseny, A.Albareda, G., Sanz, A. S., Mompart, J.,and Oriols,X. (2014). Applied Bohmian Mechanics. Euro. Phys. J. D. 68: 286.

2. Browne, W.R. and Feringa,B. L. (2006). Making molecular machines work. Nature Nanotechnology 1: 25-35.

3. Chou, C.C. and Wyatt, R. E. (2007).Quantum trajectories in complex space. Phys. Rev. A.76: 012115.

4. Cressman, A., Togashi, Y.,Mikhailov, A. S. and Kapral,R. (2008).Mesoscalemodeling ofmolecular machines: cyclic dynamics and hydrodynamical fluctuations. Phy. Rev.E. 77(5).

5. Crowell, L. B. (2005). Quantum Fluctuations of Spacetime, World Scientific Publishing Co. Re. Ltd.: Singapore.

6. Echeverria, J. and Joachim,C.(2015). The Design of a Single-Molecule Motor: Advances in Atom and Single Molecule Machines. Springer International Publishing Switzerland.

7. Eingorn, M. V. and Rusov, V. D. (2014). Emergent quantum Euler equation and Bose-Einstein condensates. Found. Physics. 44: 183-191.

8. Garashchuk, S. and Rassolov, V. A. (2002). Semiclassical Dynamics Based on Quantum Trajectories. Chem. Phys. Letters. 364: 562-567.

9. Goldtzvik, Y., Zhang, Z. and Thirumalai,D. (2016). Importance of Hydrodynamic Interactions in the Stepping Kinetics of Kinesin. J. Phys. Chem. B120(8): 2071-2075.

10. Joachim, C. and Gimzewski,J. K.(2001).Molecular Machines and Motors. Sauvage, J.P. Eds. Springer: Verlag Berlin Heidelberg New York.

11. Joachim, C. and Rapenne, G.(2015). Single Molecular Machines and Motors: Advances in Atom and Single Molecule Machines. Switzerland: Springer International Publishing.19-20 June 2013. 81-94.

12. Joachim, C., and Ratner, M. A. (2005). Molecular electronics: some views on transport junctions and beyond. Proceedings of the National Academy of Sciences of the United States. US,America. 19 January 2005. 102(25): 8801-8808.

13. Kottas, G.S., Clarke, L. I.,Horinek, D. and Michl, J. (2005). Artificial Molecular Rotors. Chem. Rev. 105: 12811376.

14. Nauenberg, M. (2014). Is Bohm's Interpretation Consistent with Quantum Mechanics.Quanta. 3(1): 43-46.

15. Schönborn, J. B., Herges, R.and Hartke,B. (2009). Brownian molecular rotors: Theoretical design principles and predicted realizations. J. Chem. Phys. 130: 234906. doi: 10.1063/1.3148223.

16. Sonego, S. (1991). Interpretation of the hydrodynamical formalism of quantum mechanics. Found. Phys.21(10): 1135-1181.

17. Tang, H., Cuberes, M. T.,Joachim C. and Gimzewski, J. K. (1997). Fundamental considerations in the manipulation of a single C60 molecule on a surface with an STM. Surf. Sci. 386(1-3): 115-123.

18. Tsekov, R. (2009). Dissipative and quantum mechanics.New Adv. Phys. 5(3): 35-44.

19. Tsekov, R. (2012). Bohmian mechanics versus Madelung quantum hydrodynamics. Ann. Univ. Sofia, Fac. Phys. Special Edition (2012) 112-119. Preprint arXiv 0904.0723

20. Wyatt, R. E. (2005). Quantum Dynamics with Trajectories; Springer: Austin, USA. 